JPPT I Position Paper

\title{
The Role of the Pediatric Pharmacist in Precision Medicine and Clinical Pharmacogenomics for Children
}

Jacob T. Brown, PharmD, MS; David Gregornik, BA, BS, PharmD; Mary Jayne Kennedy, BScPharm, PharmD, on Behalf of the Advocacy and Research Committees

With the initiatives by the National Institutes of Health and the US Food and Drug Administration, pharmacogenomics is transitioning from the laboratory to patient care. Nearly 200 drug products now contain pharmacogenomic information as part of their labeling; many of these products are commonly used in the pediatric population. Because pharmacogenomic testing can provide patient-specific predictors for drug response, pharmacists are positioned to assume a leadership role in pharmacogenomic testing, clinical interpretation of results, and recommendations for individualization of drug therapy. Opportunities for pharmacists exist in both inpatient and outpatient settings, such as pharmacist-managed clinical pharmacogenomics consultation services and educating patients and families about pharmacogenomic testing. Given the potential for genetic and age-dependent factors to influence drug selection and dosing, pediatric pharmacists should be involved in the development of dosing recommendations and interprofessional practice guidelines regarding pharmacogenomic testing in pediatric patients. Opportunities to become knowledgeable and competent in pharmacogenomics extend from coursework as part of the pharmacy curriculum to postgraduate education (e.g., residencies, fellowship, continuing education). The Pediatric Pharmacy Advocacy Group acknowledges a need for increased education of both students and practicing pharmacists with consideration for infants and children.

ABbreVIATIONS PGRN, Pharmacogenomics Research Network; PPAG, Pediatric Pharmacy Advocacy Group

KEYWORDS pediatrics; pharmacist; pharmacogenetics; pharmacogenomics; precision medicine

J Pediatr Pharmacol Ther 2018;23(6):499-501

DOI: $10.5863 / 1551-6776-23.6 .499$

\section{Background}

Patient response to medication therapy is highly variable, and adverse events in children are often unpredictable. Some patients may have significant adverse reactions to standard doses of a given drug and require a reduced dose, whereas others may require a substantially larger dose of the same drug to achieve a similar exposure and presumably an equivalent therapeutic response. Over the last 2 decades, a dramatic increase has occurred in our understanding of how drug response may be influenced by variations in a single gene (pharmacogenetics), gene networks, and/or the entire genome (pharmacogenomics). With the completion of the Human Genome (2003) and International HapMap (2007) Projects, the information and tools necessary to identify important gene-response associations are now widely available. Increased availability of genetic tests in clinical laboratories (including direct-toconsumer testing) and decreasing analytical costs are also facilitating the uptake of pharmacogenomic testing in patient care. The promise of precision medicine is therefore steadily working its way into clinical care. There is no doubt that pharmacogenomics now plays an important role in drug development, ${ }^{1-4}$ regulation, and prescribing. For pharmacists, pharmacogenom- ics is also proving to be a powerful clinical tool that, when used to develop individualized treatment plans for children, may ultimately increase the likelihood of selecting the right drug at the right dose for the right patient, reducing the risk of adverse drug events and/ or treatment failure.

The widespread clinical implementation of pharmacogenomics has admittedly been slower than anticipated following completion of the Human Genome Project; however, advancing pharmacogenomics into clinical practice remains a national healthcare priority. At the forefront of this effort are federal organizations such as the National Institutes of Health, which created and provided funding for a collaborative research network dedicated to the discovery of clinically relevant gene/ drug response associations (the Pharmacogenomics Research Network [PGRN]). As a result of PGRN-sponsored research, the Pharmacogenomics Knowledge Base, a leading, publicly available database in the field of pharmacogenomics, has been created. The Clinical Pharmacogenetics Implementation Consortium, an extension of the PGRN, was formed in 2009 to address barriers to clinical implementation and to develop and update actionable drug-gene guidelines to guide medication and dose selection based on pharmacogenomic test results. The first of these guidelines, published in 
March 2011, provides dosing recommendations for the thiopurine drugs based on the thiopurine methyltransferase genotype. ${ }^{5}$ Currently, Clinical Pharmacogenetics Implementation Consortium guidelines are available for 36 medications (cpicpgx.org/guidelines), albeit with limited pediatric-specific recommendations. ${ }^{6}$

\section{Potential Roles and Activities for Pediatric Pharmacists in Pharmacogenomics}

The roles that pharmacists play in clinical pharmacogenomics are more defined since the publication of the original position statement in 2011; however, there are still great opportunities for our profession and practice specialty to advocate for, and to establish the role of, pediatric pharmacists in pharmacogenomics. In their role as part of the multidisciplinary team, pediatric pharmacists routinely make medication and dose recommendations based on several clinical factors, including a child's age, physiology, concomitant medications, and diagnosis. Understanding a patient's pharmacogenomic information, including the ontogeny of these drug-metabolizing enzymes, transporters, and receptors, increases the likelihood of selecting a medication that is safe and effective for the individual. The pediatric pharmacist may use this knowledge to proactively reduce the risk of untoward adverse drug events while also avoiding therapeutic failures. Based on their education and training, pediatric pharmacists with a working knowledge of pharmacogenomics are arguably the most appropriate members of the medical team to recommend pharmacogenomic testing and to provide an interpretation of results in the context of a child's pharmacotherapy. Pediatric clinical pharmacists also routinely serve on pharmacy and therapeutics committees and work closely with pharmacy information technology support. In these roles they are well positioned to recommend gene/drug pairs for implementation within an institution as well as to create clinical decision support tools that are tailor-made for prescribing physicians, physician assistants, and nurse practitioners. While children pose unique challenges for pharmacogenomic interpretation, as compared to adults, successful implementation programs already exist at hospitals such as St. Jude Children's Research Hospital, Cincinnati Children's Hospital Medical Center, and Children's Minnesota.

\section{Challenges and Opportunities in Pharmacogenomics Education and Training}

The Accreditation Council for Pharmacy Education 2016 Standards include pharmacogenomics as a required element of the didactic Doctor of Pharmacy curriculum, and graduates of contemporary pharmacy programs are expected to be "practice-ready" with respect to integrating genetic information into patient- centered pharmaceutical care.? Although the number of colleges or schools including pharmacogenomics in their didactic curriculum continues to grow, pharmacogenomic instruction remains inadequate, especially in areas related to pediatric-specific topics. ${ }^{8-10}$ Opportunities for experiential and/or practice-based training within Doctor of Pharmacy programs are also uncommon, with only a few programs offering elective Advanced Pharmacy Practice Experiences in precision medicine and/or pharmacogenomics. Consequently, many practicing pharmacists lack confidence and feel inadequately prepared to apply this information in practice. ${ }^{11}$

Opportunities for formal postgraduate training in pharmacogenomics remain limited, with only 4 programs (St. Jude Children's Research Hospital, the University of Florida College of Pharmacy, Children's Minnesota, and the University of Chicago at Illinois College of Pharmacy) offering postgraduate year 2 residencies in pharmacogenomics. Fellowship and formal graduate programs are available at the master's and doctoral levels, although these types of programs are typically research focused and reserved for individuals seeking careers in academia or industry. For practitioners, there are several professional development opportunities available beyond formal postgraduate training, including traditional continuing pharmacy education programs offered live as part of the programming at national meetings or as standalone programs, many of which are self-paced and available online. Examples of such programs include the Pharmacogenomics Education Program (PharmGenEd) offered by the University of California-San Diego and the American Pharmacists Association (APhA) training course developed collaboratively with Manchester University and RxGenomix. Certificate programs provide an alternative for practitioner education that allows more in-depth exposure to clinical pharmacogenomics than a traditional continuing pharmacy education program. ${ }^{12}$ Examples of such programs include the Test2Learn program developed by the National Association of Chain Drug Stores in collaboration with the University of Pittsburgh School of Pharmacy; the Pharmacogenomics Certificate Program offered by the University of Colorado School of Pharmacy; the Pharmacogenomics and Precision Medicine Certificate offered by Shenandoah University; and the Pharmacogenomics Certificate Program offered as part of the annual Precision Medicine Conference at the University of Florida College of Pharmacy.

Some hospital systems have also developed continuing education for their pharmacists. The Children's Hospital of Wisconsin developed an online and live module course based on objectives from the National Coalition for Health Professional Education in Genetics guidelines. ${ }^{13}$ The online module includes introductory concepts on genetics and pharmacogenomics, particularly related to antiepileptic medications, and is 
a required competency for all pharmacists to provide pharmacogenomic services at the hospital. ${ }^{14}$ St. Jude Children's Research Hospital has also developed educational programs for their pharmacists to complete before providing pharmacogenomic consultation, serving as an example for other institutions to follow.

\section{Recommendations}

The Pediatric Pharmacy Advocacy Group (PPAG) endorses the involvement of pediatric pharmacists in pharmacogenomic testing and believes that pharmacists should be the healthcare professionals responsible for ordering, interpreting, and applying pharmacogenomic test results as they relate to pediatric pharmacotherapy. The PPAG also strongly encourages pharmacists to take responsibility for educating patients and their families about pharmacogenomic testing, especially in the community setting, where direct-to-consumer genetic test kits are readily available to patients and caregivers. The PPAG acknowledges a need for increased application-based education of both students and practicing pharmacists, which should incorporate clinical applications, such as interpretation of test results with consideration of infants and children. The PPAG believes that pharmacogenomics is an emerging discipline that will become increasingly important in pediatric pharmacotherapy. As genotype-based dosing recommendations are developed and updated, the need for pediatric pharmacists trained in this discipline will become essential.

\section{ARTICLE INFORMATION}

Affiliations University of Minnesota College of Pharmacy (JTB), Duluth, Minnesota, Children's Minnesota (DG), Minneapolis, Minnesota, Fred Wilson School of Pharmacy at High Point University (MJK), High Point, North Carolina

Correspondence Pediatric Pharmacy Advocacy Group; Jennifer.Chow@ppag.org

Disclosure The authors declare no conflicts or financial interest in any product or service mentioned in the manuscript, including grants, equipment, medications, employment, gifts, and honoraria.

Acknowledgments Authors on the first version were Mary Jayne Kennedy, BScPharm, PharmD; Hanna Phan, PharmD; Sandra Benavides, PharmD; Amy Potts, PharmD; and Susan Sorensen, PharmD.

Accepted September 19, 2018

Copyright Published by the Pediatric Pharmacy Advocacy Group. All rights reserved.

For permissions, email: matthew.helms@ppag.org

\section{REFERENCES}

1. US Department of Health and Human Services. Guidance for Industry: Pharmacogenomic Data Submissions. https://www.fda.gov/downloads/drugs/guidancecomplianceregulatoryinformation/guidances/ucm079849.pdf. Accessed September 19, 2018.

2. Williams JA, Andersson T, Andersson TB, et al. PhRMA white paper on ADME pharmacogenomics. J Clin Pharmacol. 2008;48(7):849-889.

3. US Food and Drug Administration. Table of Pharmacogenomic Biomarkers in Drug Labeling. https://www.fda.gov/ drugs/scienceresearch/researchareas/ pharmacogenetics/ucm083378.htm. Accessed September 19, 2018.

4. Committee on Quality of Health Care in America, Institute of Medicine. To Err Is Human: Building a Safer Health System. Washington, DC: National Academy Press; 2000.

5. Relling MV, Gardner EE, Sandborn WJ, et al. Clinical pharmacogenetics implementation consortium guidelines for thiopurine methyltransferase genotype and thiopurine dosing: 2013 update. Clin Pharmacol Ther. 2013;93(4):324-325.

6. Clinical Pharmacogenetics Implementation Consortium (CIPC) Guidelines. cpicpgx.org/guidelines. Accessed September 19, 2018.

7. Accreditation Standards and Key Elements for the Professional Program in Pharmacy Leading to the Doctor of Pharmacy Degree. Accreditation Council for Pharmacy Education. Standards 2016. https://www.acpe-accredit. org/pdf/Standards2016FINAL.pdf. Accessed September 19, 2018.

8. Latif DA, McKay AB. Pharmacogenetics and pharmacogenomics instruction in colleges of pharmacy in the United States. Am J Pharm Educ. 2005;69(2):152-156.

9. Murphy JE, Green JS, Adams LA, et al. Pharmacogenomics in the curricula of colleges and schools of pharmacy in the United States. Am J Pharm Educ. 2010;74(1):1-10.

10. Huston SA, Zdanowicz MM, Fetterman JW. Pharmacogenomics in advanced pharmacy practice experiences. $A m$ J Pharm Educ. 2010;2:196-203.

11. Tuteja S, Haynes K, Zayac C, et al. Community pharmacists' attitudes towards clinical utility and ethical implications of pharmacogenetic testing. Per Med. 2013;10(8):793-800.

12. Weitzel KW, Aquilante CL, Johnson S, et al. Educational strategies to enable expansion of pharmacogenomicsbased care. Am J Health Syst Pharm. 2016;73(23):19861998.

13. National Coalition for Health Professional Education in Genetics. Core Competencies in Genetics for All Health Professionals. 3rd ed. September 2007. http://www. nchpeg.org/index.php?option=com_content\&view=arti cle\&id=94\&ltemid=84. Accessed March 31, 2011.

14. Zembles T. An inservice program on pharmacogenetics to individualize drug therapy. Am J Pharm Educ. 2010;74(1):10. 